\title{
Síndrome de Percheron: Lesiones talámicas bilaterales
}

\author{
Salinas Vela FT. ${ }^{1}$, Arcos Sánchez C. ${ }^{2}$
}

Sanid. mil. 2014; 70 (1): 30-32; ISSN: 1887-8571

\begin{abstract}
RESUMEN
El síndrome de Percheron o infarto talámico bilateral sincrónico se considera infrecuente y de difícil diagnóstico clínico. Presentamos el caso de un paciente con lesiones isquémicas agudas en ambos tálamos y mesencéfalo anterior, compatibles con obstrucción del la arteria de Percheron. La compleja irrigación talámica y la variabilidad individual hacen que las lesiones isquémicas puedan presentarse en forma de lesiones bilaterales y confieren importancia a este cuadro. La Resonancia Magnética cerebral (RM) es fundamental en el correcto diagnóstico.
\end{abstract}

PALABRAS CLAVE: Infarto talámico bilateral. Arteria de Percheron. Síndrome de Percheron, Patología talámica.

Percheron Syndrome: Thalamic bilateral lesions

SUMMARY: Percheron Syndrome also known as bilateral synchronic thalamic infarction is consider as an infrequent of difficult diagnosis syndrome. We describe the case of a male patient presenting acute ischemic lesions in both thalamus and anterior mesencephalon caused by an obstruction of the Percheron artery. The complexity of thalamic vascularization and individual differences make ischemic lesions appear as bilateral, that's the importance of this syndrome. Brain MRI is proposed as fundamental for a correct diagnosis.

KEY WORDS: Bilateral thalamic infraction, Percheron artery, Percheron artery syndrome, Thalamic disease.

\section{INTRODUCCIÓN}

La vascularización del tálamo fue descrita de una forma más completa por Percheron en los años 60 del siglo XX, aunque otros muchos autores habían hecho ya aportaciones previas en este sentido. Como se verá en esta comunicación, la irrigación del tálamo proviene principalmente de las variadas conexiones que establecen la arteria carótida interna y la arteria basilar. Mencionaremos la importancia que tiene la conocida como arteria de Percheron y su contribución en un territorio de vascularización complicada y variable y que, ante cuadros de isquemia, la afectación en el tálamo puede presentarse en forma de lesiones bilaterales observadas en resonancia magnética (RM). Dichas lesiones son un hallazgo infrecuente y, a menudo, se manifiestan como afectación talámica bilateral, aún cuando la afectación arterial puede ser unilateral $1^{1,2}$.

\section{PRESENTACIÓN DEL CASO}

Paciente de 60 años que acude a Urgencias porque, según refiere la familia, al despertar, tras 15 horas de sueño, presenta un cuadro de desorientación, dificultad en la articulación del lenguaje, con emisión y comprensión conservadas, visión borrosa y visión doble vertical. No ha presentado pérdida de conciencia, palpitaciones, dolor torácico ni fiebre.

${ }^{1}$ Cte. Médico Servicio de Radiodiagnóstico.

${ }^{2}$ Cte. Médico Servicio de Neurología.

Hospital General de la Defensa «Orad y Gajías» de Zaragoza. España.

Dirección para correspondencia: fsalvel@fn.mde.es

Recibido: 19 de febrero de 2013

Aceptado: 5 de julio de 2013
Entre sus antecedentes destaca que se trata de un paciente exfumador desde hace 15 años, hipertenso en tratamiento con enalapril y dislipémico en tratamiento con simvastatina.

A la exploración se evidencia paresia del IV par craneal del ojo derecho, así como afectación incompleta del III par bilateral y VI par derecho de predominio inferior manifestada en forma de diplopía binocular vertical. El resto de los pares craneales son normales. Tampoco se evidencia déficit motor ni sensitivo asociados, Romberg negativo, no dismetrías y no signos meníngeos. Se evidencia alteración de la marcha con tendencia a la lateropulsión hacia la derecha.

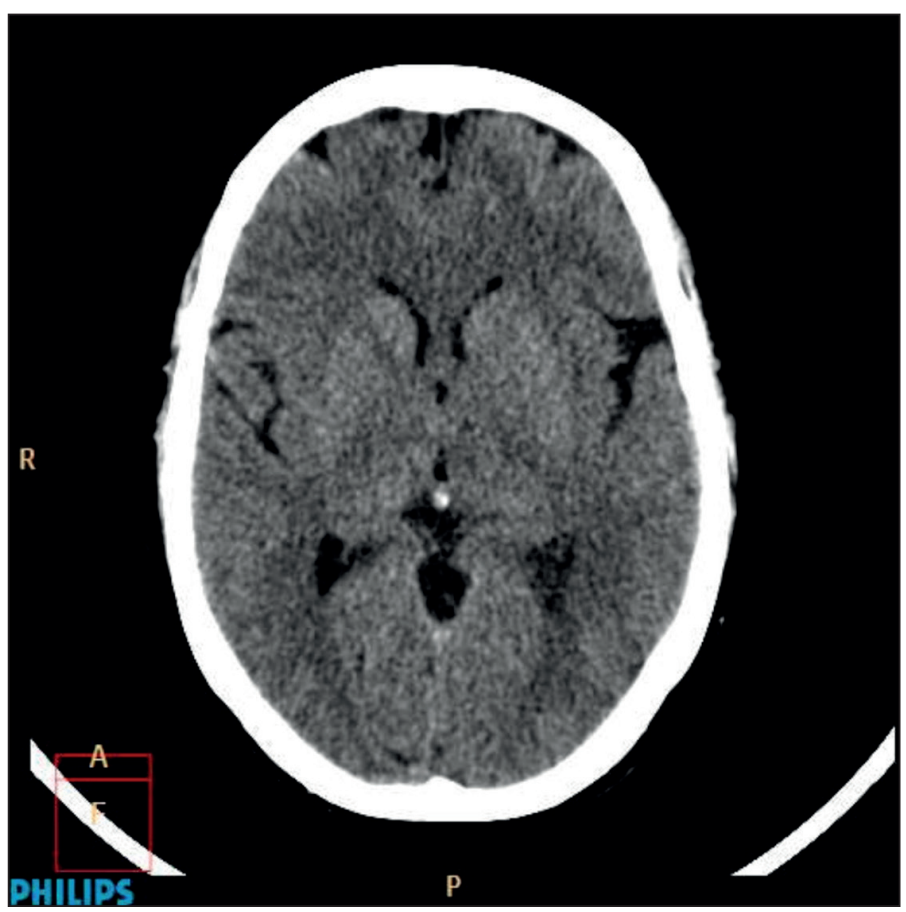

Figura 1. CT sin contraste. No se aprecian lesiones. 
Además del protocolo básico de urgencias, hemograma, bioquímica, estudio de coagulación, radiografía simple de tórax y electrocardiograma que fueron normales, se le realiza tomografía computerizada (TC) craneal de 64 canales antes del ingreso en el Servicio de Neurología, que se informa como exploración sin aparentes hallazgos patológicos (figs. 1 y 2).

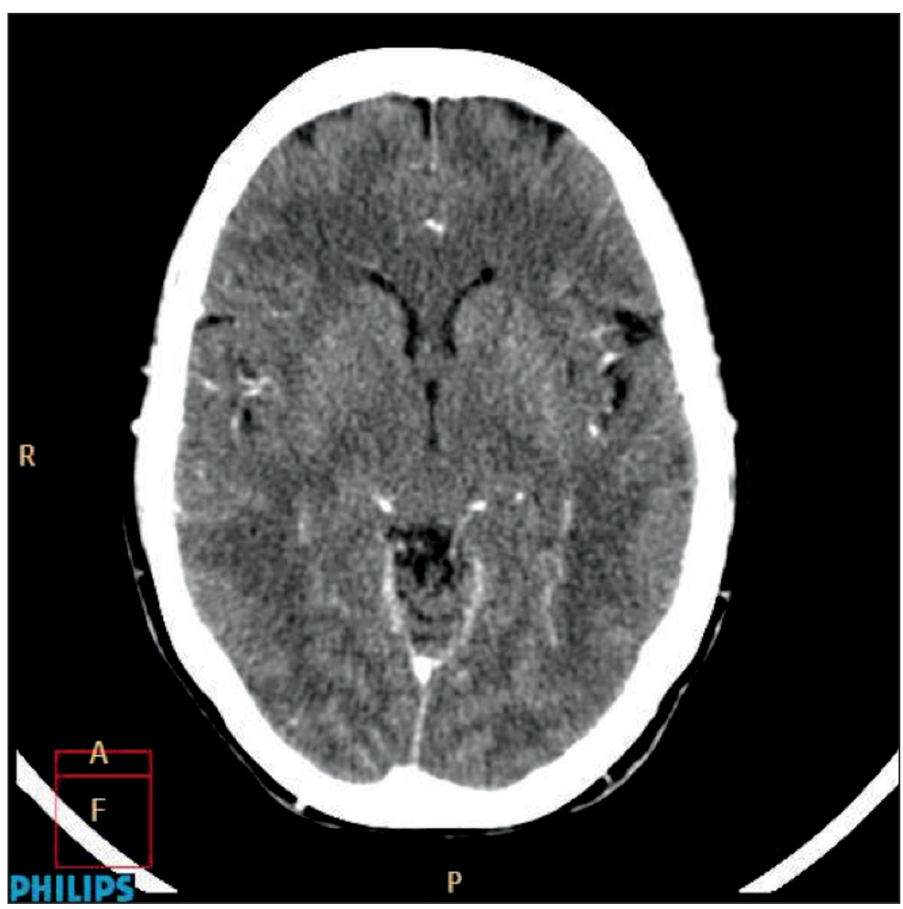

Figura 2. CT con contraste. No se aprecian lesiones.

Durante el ingreso persiste la diplopia que se corrige con oclusión monocular alternante, persistiendo la inestabilidad y el trastorno de la marcha.

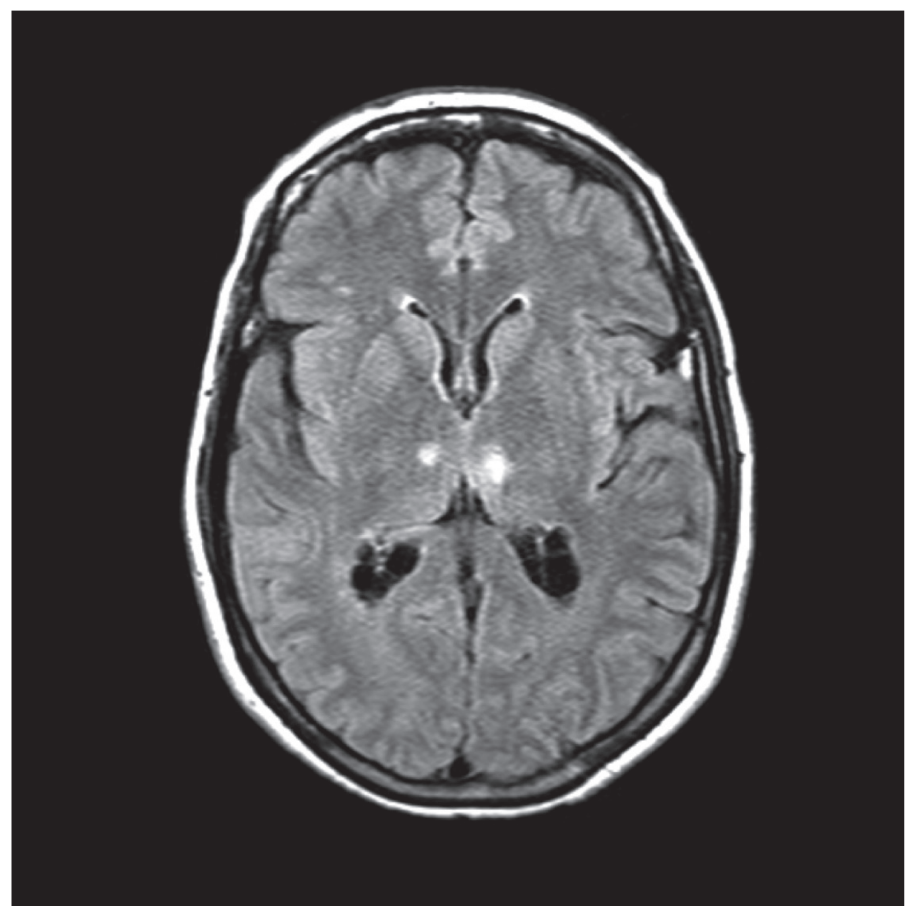

Figura 3. RM secuencia Flair. Lesiones talámicas bilaterales.
Al tercer día del ingreso se completa estudio con RM encefálica con resonancia Philips de 1,5 T, con el resultado de microinfartos solitarios en sustancia blanca profunda, así como lesiones isquémicas agudas de localización paramedial en ambos tálamos y mesencéfalo anterior, compatibles con obstrucción de la arteria de Percheron $^{3}$ (figs. 3, 4 y 5). Resultan especialmente útiles para observar estas lesiones las secuencias Flair y el uso de la Difusión.

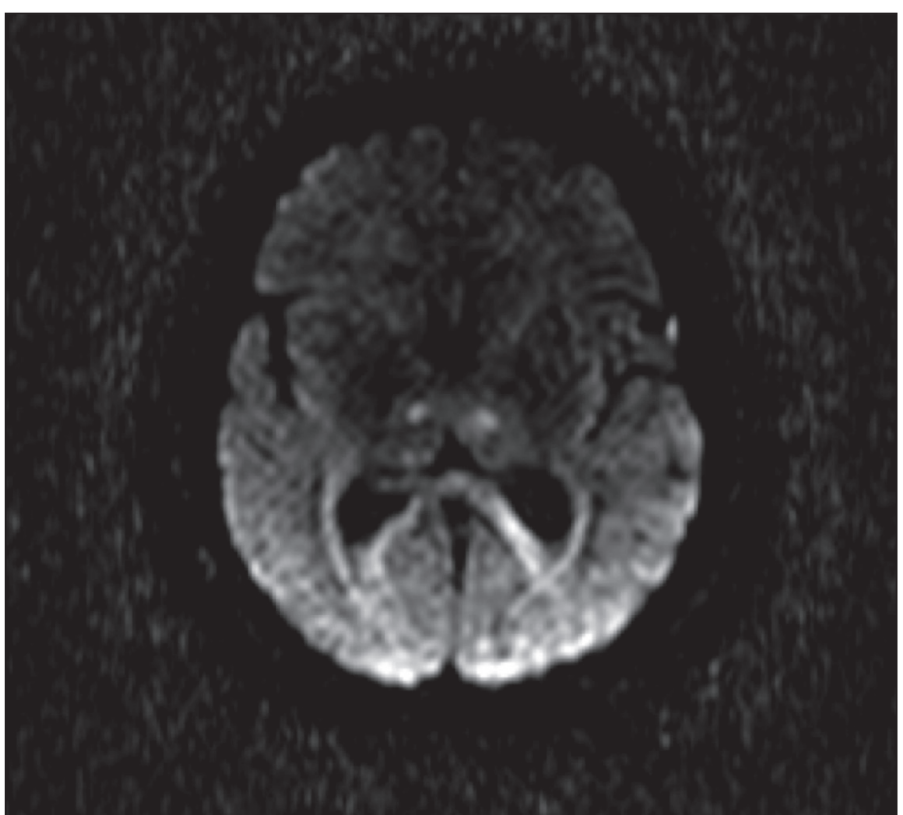

Figura 4. RM difusión. Lesiones talámicas bilaterales.

Consultado el caso con el Servicio de cardiología y, ante el origen embólico del cuadro, se decidió iniciar anticogulación oral con Dabigatran por elevado riesgo de recurrencia. La evolución de la paciente fue favorable y, con el tratamiento rehabilitador se consiguió autonomía de la marcha, presentó mejoría progresiva de la diplopía.

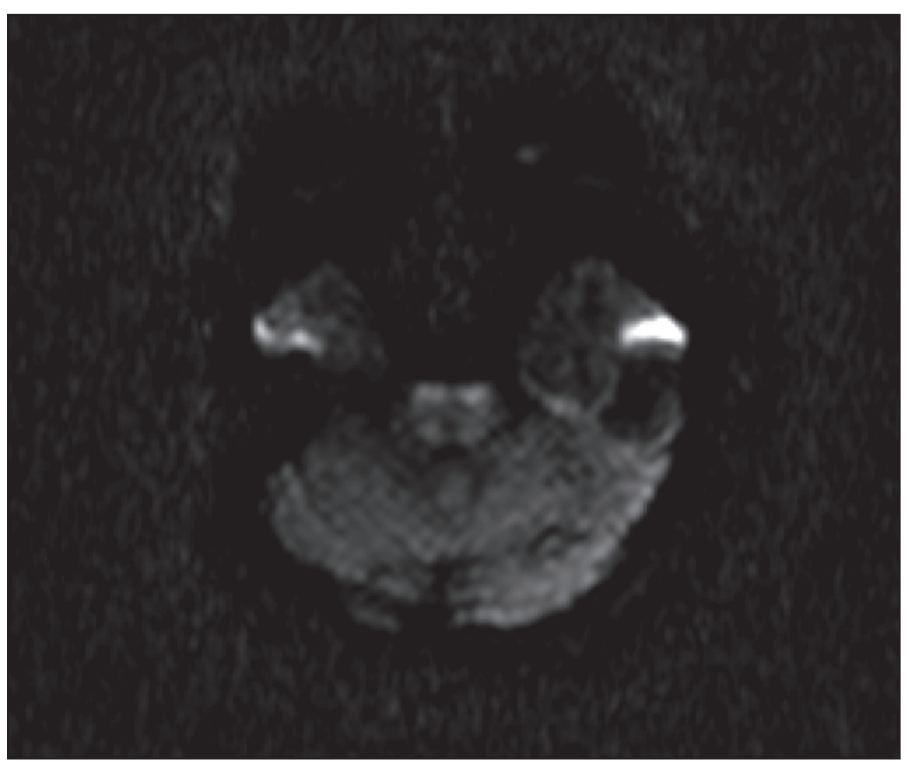

Figura 5.RM difusión. Lesiones mesencefálicas. 


\section{DISCUSIÓN}

La irrigación talámica es realizada por numerosas arterias que se originan en la base del cráneo. Son la arteria comunicante posterior y la arteria cerebral posterior las que participan principalmente en esta vascularización. Así pues, la primera porción de la arteria cerebral posterior se extiende entre el extremo superior de la arteria basilar y la salida de la arteria comunicante posterior. Percheron y otros autores denominaron a esta arteria como arteria comunicante basilar (o arteria mesencefálica), quedando el nombre de arteria cerebral posterior para la parte distal a la salida de la arteria comunicante posterior ${ }^{4-6}$.

Las arterias talámicas se originan a partir de estas estructuras (arteria comunicante basilar y arteria comunicante posterior) y son: la arteria talámica polar, la arteria talámica paramediana (o de Percheron), el pedículo tálamo geniculado y la arteria coroidal posterior con sus dos ramas posteromedial y posterolateral.

La contribución de la arteria cerebral anterior a la irrigación del tálamo a través de la arteria coroidea posterior es controvertida y, de existir, pequeña. La arteria cerebral media no participa en la irrigación del tálamo.

Una vez revisada la irrigación talámica, hay que mencionar la importancia que tiene el hecho de que estas ramas sean terminales, sin anastomosis entre ellas, para la aparición de lesiones isquémicas.

Centrándonos en la arteria paramediana de Percheron (también llamada arteria óptica interna de Duret o arteria tálamoperforante de Foix y Hillerman) hay que señalar que se origina en la arteria comunicante basilar y que Percheron observó tres formas distintas en su origen (fig. 6):

- Tipo 1: origen simétrico, bilateral ipsilateral (50\% de frecuencia).

- Tipo 2: origen asimétrico, las arterias para el tálamo derecho e izquierdo nacen de una misma arteria comunicante basilar sin preferencia de lado ( $45 \%$ de frecuencia), dividiéndose a su vez en:

- Tipo 2 A: nacen independientemente en la misma comunicante basilar.

- Tipo 2 B: nacen en un tronco común en la misma comunicante basilar.

- Tipo 3: disposición simétrica, en arcada, conectando ambas comunicantes basilares y originando dos arterias paramedianas ipsilaterales (frecuencia 5\%).

Esta arteria paramediana de Percheron, independientemente de sus distintos orígenes, irriga territorio talámico y subtalámico, a nivel de mesencéfalo ${ }^{7}$.

El resto de las arterias que irrigan el tálamo son:

- La arteria polar, originada a nivel de la unión del tercio medio con el tercio distal de la arteria comunicante posterior, en un $30-40 \%$ de la población está ausente y es sustituida por la arteria paramediana.
- El pedículo tálamogeniculado se forma a partir de cinco o seis pequeñas arterias que se originan de la arteria cerebral posterior.

- La arteria coroidal posterior se origina de la arteria cerebral posterior y presenta una rama posteromedial y otra posterolateral.

Todo esto es importante ya que las lesiones isquémicas bilaterales del tálamo, observadas en estudios de resonancia magnética son un hallazgo infrecuente. La particularidad de la circulación talámica hace posible el compromiso de ambos tálamos. Reconocer esta afectación bilateral en los estudios de RM es fundamental para caracterizar el cuadro ${ }^{8}$.

\section{CONCLUSIÓN}

Cuando tiene lugar un cuadro isquémico dependiente de la arteria de Percheron las manifestaciones neurológicas que suelen aparecer son la afectación del nivel de conciencia con cambios fluctuantes en el mismo, incluyendo el coma, y además suele existir afectación del lenguaje, así como alteración del estado de ánimo en forma de apatía, y otras manifestaciones como afectación de pares craneales oculomotores, trastornos del movimiento (disquinesias), afectación de la memoria (amnesia) y del sueño (hipersomnia). Esta forma de presentación es muy aproximada a la del caso descrito y confirma la necesidad de conocer el territorio irrigado por una arteria como la de Percheron, puesto que las lesiones que desencadena suelen ser alteraciones talámicas bilaterales.

Reconocer esta afectación bilateral en los estudios de RM es fundamental para caracterizar el cuadro; además, cabe destacar que existen pocos casos descritos de hiperintensidad bilateral en tálamo y la escasa utilidad que han demostrado otras pruebas de neuroimagen.

\section{BIBLIOGRAFÍA}

1. Bell AD, Davis WC, Osborn AG. Bithalamic hiperintensity on T2, weighted MR vascular causes and evaluation with MR Angiography. AJNR Am J Neuroradiol 1994; $15: 893-99$.

2. Smith B, Smirniotopoulos JG, Rushing EJ, Goldstein SJ. Bilateral thalamic lesions. AJR Am J Roentgenol 2009;192:53-62.

3. Matheus MG, Castillio M. Imaging of acute bilateral paramedian thalamic and mesencephalic infarcts. AJNR Am J Neuroradiol 2003; 24:2005-08.

4. Percherón G. Les artères du thalamus humain.1: artère et territoire thalamiques polaires de l'artère comunicante postérieure. Rev Neurol (Paris). 1976;132:297-307.

5. Percherón G. Les artères du thalamus humain.2: artères et territories thalamiques paramédians de l'artère basilaire communicante. Rev Neurol (Paris) 1976;132:309-24.

6. Percheron G. Les artères du thalamus humain. Les artères choroïdiennes. 1: Étude macroscopique des variations individuelles. 2: Systématisation. Rev Neurol (Paris) 1977; 133:533-45.

7. Carrera E, Michel P, Bogousslavsky J. Anteromedian, central and posterolateral infarcts of the thalamus, three variant types. Stroke 2004; 35:2826-31.

8. Hegde AN, Mohan S, Lath N, Lim CCT. Differential diagnosis for bilateral abnormalities of basal ganglia and thalamus. Radiographics 2011; 31:5-30. 